\title{
Globe
}

Revue internationale d'études québécoises

\section{Penser le lien collectif. Mémoire et identité au Québec}

\section{Jocelyn Maclure}

Volume 5, numéro 2, 2002

Penser le lien collectif

URI : https://id.erudit.org/iderudit/1000677ar

DOI : https://doi.org/10.7202/1000677ar

Aller au sommaire du numéro

Éditeur(s)

Globe, Revue internationale d'études québécoises

ISSN

1481-5869 (imprimé)

1923-8231 (numérique)

Découvrir la revue

Citer ce document

Maclure, J. (2002). Penser le lien collectif. Mémoire et identité au Québec. Globe, 5(2), 13-20. https://doi.org/10.7202/1000677ar d'utilisation que vous pouvez consulter en ligne.

https://apropos.erudit.org/fr/usagers/politique-dutilisation/ 


\title{
Introduction
}

\section{Penser le lien collectif. Mémoire et identité au Québec}

\author{
Jocelyn Maclure \\ Université de Southampton (Angleterre)
}

Quelles sont les conditions de possibilité du lien collectif à une époque marquée par l'éclatement des appartenances, la multiplication des visions du Bien et la diversité culturelle ? Comment penser l'intégration des sociétés une fois leur caractère hétérogène reconnu ? Les philosophes politiques appartenant à la tradition contractualiste, de Hobbes au premier Rawls, ont longtemps soutenu qu'il fallait imaginer les êtres humains dans des conditions pré-sociales afin de déterminer les droits et les devoirs que ceux-ci devaient se conférer mutuellement. Ces expériences de pensée ou ces fictions méthodologiques nous paraissent bien moins utiles aujourd'hui. Il s'agit plutôt maintenant de penser la communauté politique en prenant à bras-le-corps le pluralisme des valeurs, des schèmes interprétatifs et des identités qui caractérisent la plupart des sociétés contemporaines.

Penser le lien collectif se veut donc l'un des défis les plus importants auquel sont confrontées les sciences humaines et sociales. La cohésion et la coopération sociales, la stabilité politique, la solidarité, la justice et la paix dépendent ultimement de ce lien qui relie les individus à travers leurs différences. Le lien collectif pose problème d'abord parce que la communauté politique ne peut plus s'en remettre à de grandes certitudes ou à des méta-récits pour asseoir son autoritél. Le désenchantement du

1. Voir Claude Lefort, Essais sur le politique ( $x x^{e}-X X^{e}$ siècles), Paris, Seuil, 1986 et Jacques Rancière, La mésentente. Philosophie et politique, Paris, Galilée, 1995.

Jocelyn Maclure, «Penser le lien collectif. Mémoire et identité au Québec ", Globe. Revue internationale d'études québécoises, vol. $5, \mathrm{n}^{\circ} 2,2002$. 


\section{REVUE INTERNATIONALE D'ÉTUDES QUÉBÉCOISES}

monde a conféré à la délibération publique la responsabilité de l'élaboration des normes et des modes de gouvernance qui fondent le vivreensemble - une responsabilité dont il est impossible. de s'acquitter une fois pour toute puisque l'érosion des repères ultimes de la certitude a fait en sorte qu'une pluralité de perspectives sur le bien commun se confrontent sans pouvoir s'en remettre à une forme d'autorité qui ne ferait pas elle-même l'objet de controverses.

C'est ainsi que l'on s'est mis à chercher les sources de l'intégration sociale dans un contexte de modernité avancée. Certains croient que la coordination des actions et la résolution des différends dépend aujourd'hui d'une éthique du discours ou d'une conception de la raison publique qui imposent des contraintes au genre de considérations pouvant être soulevées publiquement afin de favoriser l'émergence de consensus. Un patriotisme de la constitution vient parfois compléter et consolider ces conceptions de la raison publique.

D'autres, comme Jacques Beauchemin dans ce numéro, affirment que le lien collectif doit être soutenu en amont par une identité nationale rassembleuse et synthétique. La solidarité sociale dans un contexte marqué par l'individualisme et la prolifération des identités particularistes ne pourrait se déployer que dans un lieu identitaire et politique suffisamment consensuel comme la nation. Doutant que la nation puisse encore véritablement jouer ce rôle, d'autres encore avancent plutôt que la diversité des identités, des valeurs, des traditions interprétatives et des intérêts fait en sorte que des désaccords au sujet des orientations publiques, des procédures d'argumentation, des règles constitutionnelles ou de la substance de l'identité nationale résistent au dialogue politique et rendent par le fait même hautement problématiques les approches consensualistes susmentionnées. Le lien collectif, selon cette dernière perspective, serait un produit dérivé de la participation et de la délibération publiques et n'exigerait donc pas l'élimination du désaccord ${ }^{2}$.

2. J'ai commencé à explorer le potentiel de cette approche dans "Pluralisme et démocratie *, Repères en mutation. Identité et citoyenneté dans le Québec contemporain, J. Maclure et Alain-G. Gagnon [éd.], Montréal, Québec Amérique, 2001, p. 251-268 et dans . The Politics of Recognition at an Impasse? Identity Politics and Democratic Citizenship *, Canadian Journal of Political Science, à paraître. 
L'intérêt pour le Québec dans le cadre des réflexions sur le lien collectif s'explique facilement. L'espace public québécois est en effet un laboratoire de pointe en aménagement de la diversité et en gestion du désaccord. Avec les onze nations autochtones et la minorité historique anglophone qui cohabitent avec la majorité francophone, le Québec est une société multinationale. La présence de ces premières nations pousse d'ailleurs le gouvernement du Québec à expérimenter une pratique politique de reconnaissance de la diversité et de partage de la souveraineté qui respecte de plus en plus les exigences' d'une approche postcoloniale 3 . De plus, en tant que société d'accueil qui doit augmenter son influx d'immigrants afin de pallier son faible taux de natalité, le Québec est marqué à la fois par le multiculturalisme et par l'interculturalismét. En termes de droits et d'intégration sociale, la diversité multiculturelle pose au politique des défis distincts de ceux engendrés par la présence de différentes minorités nationales.

Les contributions réunies dans ce numéro explorent toutes, explicitement ou implicitement, les nouvelles formes du vivre-ensemble en contexte de diversité profonde 5 . Plus précisément, la nécessité de penser à nouveaux frais la relation entre les Québécois d'héritage canadien-français et les Québécois anglophones et d'origine immigrante unit ces différentes analyses tel un fil d'Ariane ${ }^{6}$. L'examen analytique et critique des questions se rapportant à la socialité au Québec exige une exploration à la fois de l'imaginaire social et des modes de régulation

\footnotetext{
3. Je pense ici aux négociations qui ont mené à la révision de l'entente de la Baie-James et au processus de négociation présentement en cours avec certaines communautés Innus.

4. Pour la différence entre ces deux phénomènes sur le plan identitaire, voir Sherry Simon, Hybridité culturelle, Montréal, L'île de la tortue, 1999 et, sur le plan de la gestion de la diversité, Alain-G. Gagnon, "Plaidoyer pour l'interculturalisme ", Possibles, vol. 24, $\mathrm{n}^{\circ} 2,2000$, p. 11-25.

5. À cet égard, ce numéro thématique de la revue Globe vient compléter le recueil de textes Repères en mutation, op. cit.

6. La dimension multinationale du Québec, c'est-à-dire la place de la minorité nationale anglophone et des nations autochtones, n'est abordée que de façon tangentielle dans les textes qui constituent ce dossier. On consultera à cet égard James Tully, "Liberté et dévoilement dans les sociétés multinationales ", Globe, vol. $2, n^{\circ} 2,1999$, p. 13-36.
} 
civique. Les mécanismes et les soubresauts du lien collectif sont à retracer tant dans les représentations de soi, le discours social et les relations civiques que dans les positions officielles de l'État québécois. Les textes regroupés ici s'attardent tous à l'une ou à plusieurs de ces dimensions du lien social.

Ce numéro thématique s'ouvre avec un essai critique décapant signé par Simon Harel. Certains commencent avec raison à penser que la critique des essentialismes de tout acabit ne suffit plus. Nul doute qu'il faille rester vigilant face aux tentatives toujours renouvelées de laminage et d'épinglage des identités. Il n'en demeure pas moins qu'il est maintenant devenu relativement aisé de s'en tenir à la posture déconstructive de la critique des essentialismes. Cette posture critique s'est muée chez certains en une apologie du nomadisme, de la fragmentation, de la transculturalité et du métissage. Harel, revenant sur certains des mouvements de pensée qui ont ponctué la réflexion sur la littérature "nationale " et, surtout, celle issue "des communautés culturelles" depuis les années 1980 , suggère que l'éloge du mouvement et de l'hybridité est aujourd'hui un réflexe de la pensée et une " doxa rassurante ". Alors qu'à l'origine cet intérêt pour la déterritorialisation se voulait un mode de problématisation du grand récit national littéraire, il serait maintenant devenu un lieu commun de la pensée sur l'identité et la littérature québécoises. Lorsque ce grand récit national fut remis en question, la valorisation des perspectives venant de la marge permit "d'édifier la littérature québécoise sur des images de tolérance, d'ouverture à l'autre . Or les écrivains migrants, aiguillonnés par leur subjectivité composite, écrivent, créent, mais ne peuvent s'acquitter de l'impossible tâche de redonner au grand récit littéraire une cohérence et une légitimité perdues. "La littérature n'est jamais l'expression d'un ethos communautaire ", nous dit Harel. Et il ajoute du même souffle : "La littérature migrante ne peut correspondre à un discours lénifiant qui propose une réunification parcimonieuse des cultures... Elle n'est pas cette agréable aporie postmoderne qui permet à tout un chacun de se dire vivre partout et nulle part . " Entre le ressassement de l'identité et la valorisation de la déterritorialisation ", demande Harel, "n'y a-t-il pas précisément "quelque chose" qui a été laissé pour compte et qui appartient à l'espace de la communauté impossible, du littéraire?" Les concepts du lieu, de l'attachement et de la sédentarité 
doivent maintenant réapparaître dans notre réflexion au côté de ceux du hors-lieu, du mouvement et de l'hybridité.

Jocelyn Létourneau explore et développe quant à lui l'idée voulant que la langue française au Québec soit à la fois une pierre d'achoppement $e t$ une condition de possibilité de la régénération des références identitaires d'un Québec soumis aux transformations de son imaginaire collectif et de son tissu social. C'est parce que la langue est autant "un lieu de mémoire " qu'un vecteur de " passage identitaire " qu'elle est à la fois impasse et possibilité. La langue, porteuse d'une mémoire, marque une distinction entre les Québécois d'héritage canadien-français et les autres Québécois. En deçà du statut clairement civique de la citoyenneté québécoise, dans la vie sociétale, un Québécois francophone d'origine non canadienne-française, trahi par son accent, est mis en position d'extériorité par rapport à la norme linguistique définie par le groupe majoritaire, ce qui ne veut pas dire selon Létourneau qu'il connaîtra la marginalisation et encore moins l'ostracisme. De façon plus profonde, l'expérience d'une langue et de la façon de la dire se réfèrent ellesmêmes à une mémoire, à une narration du passé qui vise autant à développer une intelligence du présent que des projets pour l'avenir. Or, Létourneau nous rappelle qu'il ne suffit pas d'être un locuteur francophone pour partager le ou les romans mémoriels de la majorité. Cela pourrait sans doute expliquer la très préoccupante tendance chez une partie des nationalistes québécois à s'inquiéter non pas du déclin du nombre de locuteurs francophones, puisque la loi 101 a radicalement changé la donne en matière d'intégration linguistique chez les nouveaux arrivants, mais de la diminution de l'influence des Québécois d'origine canadienne française sur l'île de Montréal et, par le fait même, au Québec.

Il ne faut toutefois pas croire que ces cloisons symboliques et linguistiques s'avèrent parfaitement hermétiques. Les rapports sociaux au Québec mettent en scène des zones de contact, de médiation et de passages entre les identités, les mémoires et les visions. Différentes pratiques, esthétiques et artistiques par exemple, serviraient à dissocier les liens trop étroits entre l'espace, la langue et la mémoire. Il est difficile, nous dit Létourneau, de prévoir si cette pratique de la médiation et de 


\section{REVUE INTERNATIONALE.D'ÉTUDES QUÉBÉCOISES}

la traduction mènera à une régénération en profondeur des référents identitaires québécois, mais au moins elle permet d'entrevoir le rapport entre langue et identité sous un jour différent.

Jutta Stamer nous offre quant à elle une lecture croisée des débats portant sur la citoyenneté et l'intégration au Québec et en Allemagne. La comparaison peut surprendre : quoi de plus éloigné du Québec et de son nationalisme affirmationniste qu'une Allemagne encore marquée par la période 1933-1945 et soucieuse de se distancier de toute manifestation nationaliste ? Cet éloignement, comme en témoigne le texte de Stamer, ne saurait masquer des similitudes. Soumise à une immigration de plus en plus intense qui vient s'ajouter à la présence' des Gastarbeiter turcs, l'Allemagne doit elle aussi trouver une façon originale de reconnaître les différences culturelles tout en assurant la cohésion de son système de régulation sociale. Le Québec offre à cet égard des pistes de réflexions susceptibles d'éclairer ce débat allemand encore en germe. Alors que l'Allemagne semble vouloir mettre de l'avant une "conception dénationalisée de la culture politique*, vaguement inspirée du patriotisme constitutionnel défendu par Jürgen Habermas et qui pourrait ouvrir la porte à la fragmentation culturelle de la société allemande, le Québec développe un modèle de culture publique commune qui vise à la fois à assurer les droits fondamentaux, à faire la promotion de la langue française, à reconnaître la diversité culturelle et à favoriser l'interculturalisme. La voie explorée par le Québec n'exige donc pas une séparation nette entre le culturel et le politique. Le Québec offre un modèle d'intégration intéressant malgré ses limites et ses impasses puisqu'il tente de concilier les impératifs du libéralisme, les objectifs légitimes de la majorité francophone et la reconnaissance de la différence.

Plusieurs ont pronostiqué au cours des dernières années la disparition du citoyen et l'érosion du lien collectif. En évoquant des phénomènes comme la mondialisation, l'effritement des souverainetés nationales, la consommation de masse et la montée de l'individualisme, certains ont avancé que les sources du lien collectif s'étaient asséchées et que les sociétés souffriraient en conséquence de plus en plus de problèmes de cohésion et d'intégration sociales. Denise Helly a mené une enquête qualitative auprès de 84 Montrêalais d'origines diverses afin 
de mesurer leur lien à la société québécoise et/ou à la société canadienne. Les résultats obtenus n'ont pas confirmé les craintes exprimées dans la littérature sur le sujet. Au contraire, ce qui ressort de l'enquête de Helly n'est pas que les fondements du vivre-ensemble se sont écroulés, mais bien qu'ils se sont multipliés. Alors que la qualité de la vie urbaine et de la socialité à Montréal, la culture des droits de la personne et le filet de protection sociale québécois font consensus auprès des personnes interrogées, d'autres fondements du lien collectif ne sont partagés que par des sous-groupes de l'échantillon : une appartenance primordiale à un Québec francophone politiquement autonome, la reconnaissance ou non des groupes culturels au sein même du Québec, une identification à la fois au Québec et au Canada et, de façon moins courante, un attachement à l'ancienne identité canadienne-française (chez trois répondants), une allégeance unique accordée au Canada et une appartenance exclusive au pays d'origine. Ces résultats montrent bien que les Québécois d'origine canadienne-française tout comme les Québécois issus de l'immigration ne déclinent pas leur appartenance au Québec et au Canada de la même façon et qu'il est fort abusif de jouer " la majorité francophone " contre les " communautés culturelles " comme s'il s'agissait de blocs homogènes et hermétiques.

Surplombant d'une certaine façon les quatre premiers textes, la contribution de Jacques Beauchemin pose directement la question de la place de la mémoire franco-québécoise dans la configuration du Québec contemporain, par le biais d'une lecture critique des réflexions de Gérard Bouchard et de Jocelyn Létourneau. Au-delà des désaccords qui les opposent, les deux historiens n'arriveraient pas à faire une juste place à la mémoire des Québécois d'héritage canadien-français. Or, le défi auquel le Québec est confronté selon Beauchemin est de trouver un port d'arrimage avec le pluralisme identitaire qui caractérise maintenant la réalité québécoise et les aspirations légitimes de la communauté majoritaire francophone qui s'enracinent dans une trajectoire historique particulière. De façon plus générale, Beauchemin considère que " le défi qui confronte les sociétés contemporaines consiste pour elles à reconstituer un horizon éthico-politique sur lequel la société pourrait se reconnaitre comme totalité et comme projet éthique de mise en commun. Le rassemblement autour de la majorité francophone pourrait selon 


\section{REVUE INTERNATIONALE D'ÉTUDES QUÉBÉCOISES}

Beauchemin servir de pierre de touche à cet effort de " retotalisation " du sujet politique québécois.

Ces textes constituent donc autant de tentatives de compréhension et d'explicitation des rouages du lien collectif. Ils témoignent à la fois de la pertinence de l'étude du cas québécois en matière de lien social et de la vitalité de la réflexion québécoise sur ce même sujet. 